\title{
Endovascular Treatment of Cavernous Sinus Dural Arterio- venous Fistula via Radial Artery and Median Cubital Vein
}

\author{
Wen Nian Tan, $\mathrm{MBBS}^{1}$, Arvin Rajadurai, $\mathrm{MBBS}^{1}$, Dhayal Balakrishnan, $\mathrm{MBBS}^{2}$ \\ ${ }^{1}$ Department of Radiology, Hospital Sungai Buloh, Selangor, Malaysia \\ ${ }^{2}$ Department of Radiology, Sarawak General Hospital, Kuching, Malaysia
}

\begin{abstract}
Cavernous sinus dural arteriovenous fistula (CS-DAVF) is an arteriovenous shunt where there is fistulous blood flow from the dural arteries from the internal or external carotid artery into the cavernous sinus. The current mainstay of therapy is endovascular treatment. We present a case of restrictive type of CS-DAVF in a 75-year-old male who presented with right eye symptoms. He was treated with embolisation using trans-radial artery access for angiographic runs and a median cubital vein access navigating into the cavernous sinus for coil deployment. This technique completely avoids the conventional technique of a femoral approach and confines all access to the arm. Therefore, there are less risks and complications associated with an arm access, improves patients' comfort and mobility post procedure. Transradial artery and cubital vein access allows for a safe and convenient alternative technique using the arm as compared with conventional transfemoral approach for treatment of CS-DAVF.
\end{abstract}

Key Words: Cavernous sinus dural arteriovenous fistula; Endovascular treatment; Transradial approach; Transvenous; Median cubital vein; Embolization

\section{INTRODUCTION}

Cavernous sinus dural arteriovenous fistula (CS-DAVF) is an arteriovenous shunt where there is fistulous blood flow from the dural arteries from the internal carotid artery (ICA) or external carotid artery (ECA) into the cavernous sinus.' Initial non-invasive radiological diagnostic imaging uses computed tomography (CT), magnetic resonance imaging (MRI), and CT/MR angiography to look for asymmetrical cavernous sinus enlargement or dilatation of draining veins. ${ }^{2}$ The gold standard imaging modality for diagnosis is digital subtraction angiography, which is also used for classification and plan- ning of treatment. ${ }^{3,4}$ In Suh et al. ${ }^{4}$, CSDAVF was classified into 3 angiographic patterns, which are proliferative, restrictive, and late restrictive types, where the venous pattern in the restrictive type shows retrograde flow into the superior and inferior ophthalmic vein with or without cortical venous reflux. There is also occlusion of normal antegrade flow into the inferior petrosal sinus. Presenting symptoms are also divided into 4 patterns (orbital, cavernous, ocular, and cerebral) based on the venous flow pattern where more aggressive ocular and cerebral patterns require closer follow-up and intervention to prevent vision loss, bleeds, or infarct neurological deficits. ${ }^{4}$

\section{Correspondence to: Wen Nian Tan, MBBS \\ Department of Radiology, Hospital Sungai Buloh, Sungai Buloh, Selangor 47000, Malaysia \\ Tel: +603-6145-4333 \\ Fax: +603-6145-4222 \\ E-mail:wennian.tan@gmail.com}

Received: March 22, 2021

Revised: May 18, 2021

Accepted: May 25, 2021

\section{Copyright (c) 2021 Korean Society of Interventional Neuroradiology \\ This is an Open Access article distributed under the terms of the Creative Commons Attribution Non-Commercial License (http://creativecommons.org/licenses/by-nc/4.0) which permits unrestricted non-commercial use, distribution, and reproduction in any medium, provided the original work is properly cited.}

pISSN 2093-9043 eISSN 2233-6273 
First-line management for CS-DAVF is endovascular embolization with the aim to occlude the fistula and maintain normal intracranial blood flow. This can be achieved via transarterial access, transvenous access, or both depending on the classification and angiographic characteristics of the fistula. 2,3,5,6 Transvenous embolization using coils or liquid embolic agents is preferred for CS-DAVF due to a higher success rate and lower risk of embolic reflux. ${ }^{3,5}$

Currently, the conventional transarterial and transvenous access for endovascular treatment of CS-DAVFs is via femoral punctures in the groin. However, we are seeing the radial approach gaining more popularity among neurointerventionists. Confining both arterial and venous access to the upper limb as compared to the groin also has several advantages. We describe a case of restrictive CS-DAVF where endovascular access was confined to the arm via the radial artery and medial cubital vein punctures.

\section{CASE REPORT}

A 75-year-old male with underlying dyslipidemia, hypertension, granuloma annulare, and gout presented to the emergency department of a countryside hospital with a 1-week history of right eye pain, periorbital swelling, diplopia, and headache. He had no preceding history of trauma. On examination, he had right eye proptosis, chemosis, subconjunctival hemorrhage, and right sixth cranial nerve palsy (Fig. 1A). His right-sided intraocular pressure was increased measuring $22 \mathrm{mmHg}$, whereas the left was normal measuring $12 \mathrm{mmHg}$. He was suspected to have a CS-DAVF, and $\mathrm{CT}$ angiography showed a dilated right superior ophthalmic vein. He was then referred to a tertiary center for diagnostic cerebral angiography. Digital subtraction cerebral angiography revealed a restrictive type where there was an early filling of contrast media into the cavernous sinus from the meningohypophyseal branches of bilateral ICAs as well as maxillary branches of bilateral ECAs (Fig. 1E). The main supply of the CS-DAVF was from the right internal and bilateral external carotid arteries. There was retrograde venous drainage into the superior ophthalmic vein with no evidence of cortical vein reflux. The normal antegrade flow into the inferior petrosal sinus was obliterated. He underwent endovascular embolization under general anesthesia via trans-radial artery access for angiographic runs and a median cubital vein puncture for transvenous access (Fig. 1B-D).
For the arterial access, a $5 \mathrm{Fr}$ Avanti introducer radial sheath (Cordis, Miami Lakes, FL, USA) was inserted into the right radial artery. After sheath insertion, 2,500 IU of heparin and $2.5 \mathrm{mg}$ of verapamil were administered intra-arterially through the side-port of the introducer. A 5 Fr Impress Sims 2 catheter $100 \mathrm{~cm}$ (Merit Medical, South Jordan, UT, USA) was navigated with an 0.035 inch Terumo guidewire (Terumo, Tokyo, Japan) into the right ICA for angiographic runs.

For transvenous access, the right medial cubital vein was punctured with an $18 \mathrm{G}$ cannula under ultrasound guidance. Subsequently, a $5 \mathrm{Fr}$ Avanti introducer radial sheath (Cordis) was inserted. A $4 \mathrm{Fr}$ Tempo Vertebral catheter $100 \mathrm{~cm}$ (Cordis) was navigated into the right facial vein. Thereafter, an $\mathrm{SL}$ Excelsior 1018 microcatheter (Stryker Neurovascular, Cork, Ireland) with a Transcend 0.014 inch guidewire (Boston Scientific, Heredia, Costa Rica) was navigated into the right superior ophthalmic vein and finally into the cavernous sinus for coils deployment. A total of 5 coils were deployed, 3 Target 360 detachable coils (Stryker Neurovascular) and 2 Nester pushable coils (Cook, Bloomington, IN, USA). Post embolization, there was no abnormal flow into the cavernous sinus or right superior ophthalmic vein (Fig. 1F). The flow into the intracranial circulation was also preserved with no perfusion defect.

The following day, clinical examination showed improvement of symptoms with normalization of right intraocular pressure measuring $14 \mathrm{mmHg}$, and he was discharged well.

During his 2-week outpatient follow-up, ocular symptoms had completely resolved with no right eye swelling, diplopia, or pain. Follow-up MRI imaging after 1 month revealed coils in-situ with no flow signal within the right superior ophthalmic vein and cavernous sinus.

\section{DISCUSSION}

Transfemoral arterial and venous access has been a conventional approach for neuro-endovascular procedures and has been the preferred choice amongst practicing clinicians. However, with the safety and benefits proven from recent neurointerventions, radial artery access has now become increasingly popular in diagnostic and therapeutic neurointerventional procedures. Current practices for radial access in the field include carotid artery stenting, embolization of aneurysm, mechanical thrombectomy, tumor embolization, and more?

A recent systemic review by Joshi et al. ${ }^{7}$ of 21 studies com- 
prising 1,342 individuals revealed that transradial access (TRA) for neurointerventional procedures has low complication rates. Major and symptomatic complications occurred in only $0.15 \%$ of patients, and minor complications occurred in $2.75 \%$ of patients. The most common complications for TRA were asymptomatic radial artery occlusion with an occurrence of $1.88 \%$. Access site hemorrhagic complications were minor and occurred in $0.47 \%$ of patients, with none of the patients requiring blood transfusion or further interventions. The transfemoral approach reported a much higher rate of access site hemorrhagic complications of $7.9 \%$. The procedural failure rate of neurointerventional procedures that require crossover to a transfemoral approach from TRA is 2 to $4.77 \%{ }^{8,9}$ This crossover is due to unsuccessful catheteri-
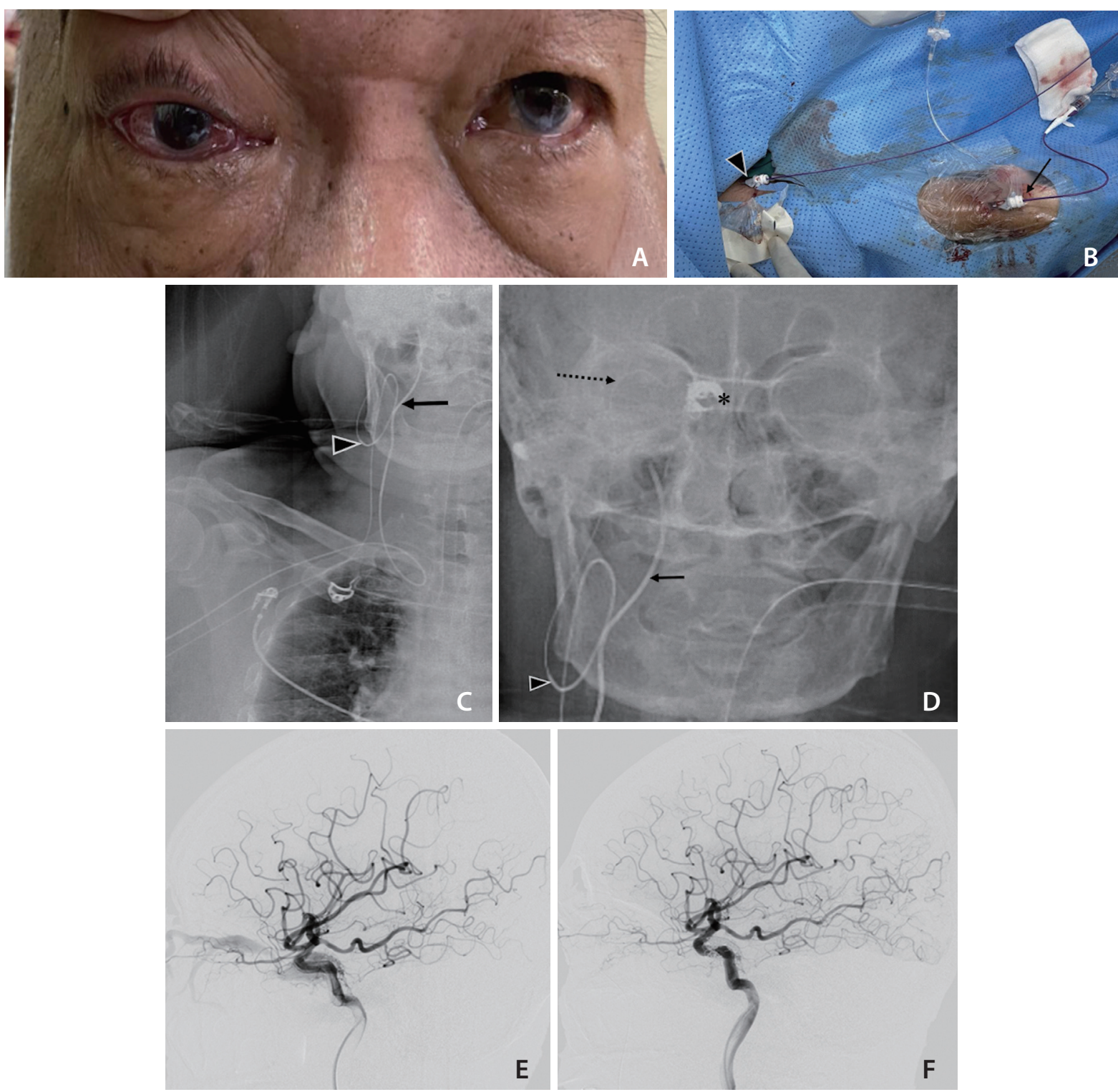

Fig. 1. (A) Pre-operative clinical examination shows presence of right eye proptosis, chemosis, and subconjunctival hemorrhage. The (B) shows upper limb arterial and venous access for transvenous embolization. Trans-radial artery access by puncture of the right radial artery (arrow) and transvenous access by puncture of the right median cubital vein (arrowhead). (C, D) 5 Fr Sims 2 catheter (Merit Medical, South Jordan, UT, USA) within the right internal carotid artery for angiographic runs (arrows). Tip of vertebral catheter at the right facial vein (arrowhead) and microcatheter further navigate into right superior ophthalmic vein and cavernous sinus (dashed arrow) with coils (asterisk) within. (E, F) Digital subtraction angiography of right internal carotid artery (ICA) in lateral view. (E) Pre embolization image shows early filling of contrast media into the cavernous sinus and superior ophthalmic vein from meningohypophyseal branches of right ICA. (F) Post embolization, coils seen within the cavernous sinus and no abnormal flow into the cavernous sinus or superior ophthalmic vein. 
zation of target vessels and severe radial artery spasm, which is comparable to a procedural failure rate of $4.7 \%$ in coronary interventions. In patients who previously experienced TRA and the transfemoral approach for neurointerventional procedures, $67-96 \%$ of patients preferred a radial approach due to the shorter recovery time and better patient comfort. ${ }^{8,10}$ Cost is also reduced with reduced complication rates and a shorter hospital stay."1

The aim of endovascular treatment in CS-DAVFs is to occlude the fistula with preservation of normal blood flow through the ICA. ${ }^{2,5}$ This can be done via the arterial route, venous route, or both. In our case, we used a regular radial artery puncture over the radial styloid with a $5 \mathrm{Fr}$ arterial sheath. A low-profile catheter (4-5 Fr) can be used with the sole purpose of performing angiographic runs and mapping the transvenous route. However, it is also possible to perform intervention from here as the radial artery over the radial styloid is usually large enough to accommodate a 6 Fr arterial sheath that will facilitate a guiding catheter needed for facilitating treatment. ${ }^{12}$ It is also feasible to place a detachable balloon via this access in cases of a traumatic carotico-cavernous fistula, as the minimum delivery guiding system is a 6 Fr catheter.

Currently in our practice, we are slowly shifting to a radial first approach in most diagnostic and selected therapeutic neurointerventional procedures. For future similar cases and purposes, we hope to move to a distal transradial artery access over the snuff box that can accommodate a 4-5 Fr sheath and reduce the risk of radial artery occlusion.

Commonly, transvenous access in neurointerventional procedures, such as mechanical thrombectomy in cerebral sinus thrombosis, inferior petrosal sinus sampling, and dural arteriovenous fistula, is through puncture of the common femoral vein. ${ }^{13}$ Alternative access is required when the transfemoral approach is not feasible in patients with local groin infections, bilateral lower limb thrombosis, inferior vena cava filter, dialysis catheter with exhausted venous access, and central vein stenosis. Apart from the common femoral vein, the internal jugular vein, superior ophthalmic vein, and superficial veins of the upper limb (basilic, cephalic, and median cubital veins) are alternative routes for venous site access.

The median cubital vein over the antecubital fossa is an easily accessible and palpable vessel that drains into the basilic vein. The basilic vein has a straighter course and larger diameter compared to the cephalic vein that forms a sharp angle when draining into the axillary vein. There may also be difficulty in passing the cephalic vein when it pierces the clavi-pectoral fascia. This makes the median cubital or basilic vein a better choice of cannulation site in superficial upper limb veins. ${ }^{11,14}$

Our approach was to confine both arterial and venous access sites to one arm. The advantage of median cubital vein access is the superficial location that facilitates easier compression if bleeding occurs as compared with transfemoral and transjugular accesses. This situation is particularly crucial in cases where patients are obese and on any form of anticoagulation or antiplatelet therapy. Another potential complication from a venous puncture in the groin or neck is an inadvertent arterial puncture and fistula formation due to the overlapping location of the underlying artery and vein. This complication can be avoided in upper limb access due to the superficial palpable nature of the median cubital vein. Other uncommon but serious adverse events in transfemoral or transjugular venous approaches include pneumothorax, hematoma formation compromising the airway, and plexus injury, all of which can be avoided if performing a superficial upper limb veins access. ${ }^{11}$ In a multicenter case series by Abecassis et al. ${ }^{15}$, patients who underwent superficial upper limb veins for neuro-interventional procedures had no adverse complications, with only $3.4 \%$ of the patients requiring conversion to the transfemoral venous approach.

There are no major technical difficulties in cannulating the jugular and facial veins from an arm approach. The guidewire is easily directed from the subclavian vein upwards into the jugular vein. From the technical aspect, both arterial and venous access catheters are straight across the arm board and do not cross or pose difficulties during the procedure. Should a left-sided access be required, bringing the arm across the body of the patient is a feasible option. The only likely scenario where an upper limb transvenous approach is contraindicated would be in patients with arteriovenous fistula in dialysis patients or patients who have an indwelling peripherally inserted central catheter.

All in all, we demonstrate a feasible, easy technique for performing embolization for spontaneous CS-DAVFs with transradial access and median cubital vein punctures for transvenous embolization. By using this technique, it completely avoids the conventional groin punctures and confines all access to the arm. Therefore, there are fewer risks and complications associated with arm access when compared to femoral access, with improved patient comfort and mobility post-procedure. 
Transradial artery and superficial upper limb vein access allows for a safe and convenient alternative technique using the arm as compared with the conventional groin approach for endovascular access in the treatment of cavernous sinus dural arteriovenous fistulas.

\section{Fund}

None.

\section{Ethics Statement}

Informed consent was taken from patients for publication of this report. Study for this case report was approved by local Institutional Review Board.

\section{Conflicts of Interest}

The authors have no conflicts to disclose.

\section{Author Contributions}

Concept and design: WNT, AR, and DB. Analysis and interpretation: WNT and AR. Data collection: AR and DB. Writing the article: WNT and AR. Critical revision of the article: WNT and AR. Final approval of the article: AR. Overall responsibility: WNT, AR, and DB.

\section{ORCID}

Wen Nian Tan: https://orcid.org/0000-0003-4365-0655 Arvin Rajadurai: https://orcid.org/0000-0002-0407-0407 Dhayal Balakrishnan: https://orcid.org/0000-0002-5909-3710

\section{REFERENCES}

1. Hiramatsu M, Sugiu K, Hishikawa T, Nishihiro S, Kidani N, Takahashi Y, et al. Results of 1940 embolizations for dural arteriovenous fistulas: Japanese Registry of Neuroendovascular Therapy (JR-NET3). J Neurosurg 2020;133:166-173

2. Gupta AK, Purkayastha S, Krishnamoorthy T, Bodhey NK, Kapilamoorthy TR, Kesavadas C, et al. Endovascular treatment of direct carotid cavernous fistulae: a pictorial review. Neuroradiology 2006:48:831-839

3. Korkmazer B, Kocak B, Tureci E, Islak C, Kocer N, Kizilkilic O. Endovascular treatment of carotid cavernous sinus fistula: a systematic review. World J Radio/ 2013;5:143-155

4. Suh DC, Lee JH, Kim SJ, Chung SJ, Choi CG, Kim HJ, et al. New concept in cavernous sinus dural arteriovenous fistula: correlation with presenting symptom and venous drainage patterns. Stroke 2005;36:1134-1139

5. Ellis JA, Goldstein H, Connolly ES Jr, Meyers PM. Carotid-cavernous fistulas. Neurosurg Focus 2012;32:E9

6. Leone G, Renieri L, Enriquez-Marulanda A, Dmytriw AA, Nappini S, Laiso A, et al. Carotid cavernous fistulas and dural arteriovenous fistulas of the cavernous sinus: validation of a new classification according to venous drainage. World Neurosurg 2019;128:e621-e631

7. Joshi KC, Beer-Furlan A, Crowley RW, Chen M, Munich SA. Transradial approach for neurointerventions: a systematic review of the literature. J Neurointerv Surg 2020;12:886-892

8. Snelling BM, Sur S, Shah SS, Khandelwal P, Caplan J, Haniff R, et al. Transradial cerebral angiography: techniques and outcomes. J Neurointerv Surg 2018;10:874-881

9. Zussman BM, Tonetti DA, Stone J, Brown M, Desai SM, Gross BA, et al. Maturing institutional experience with the transradial approach for diagnostic cerebral arteriography: overcoming the learning curve. J Neurointerv Surg 2019;11:1235-1238

10. Khanna O, Sweid A, Mouchtouris N, Shivashankar K, Xu V, Velagapudi $L$, et al. Radial artery catheterization for neuroendovascular procedures. Stroke 2019;50:2587-2590

11. Galvan Fernandez J, Martínez-Galdámez M, Schüller Arteaga M, Ortega-Quintanilla J, Hermosín A, Crespo-Vallejo E, et al. Arm-only access for combined transarterial and transvenous neurointerventional procedures. J Neurointerv Surg 2021;13:3941

12. Weinberg JH, Sweid A, Hassan A, Tekle W, Sajja K, Thaete L, et al. Early experience with a novel 088 long sheath in transradial neurointerventions. Clin Neurol Neurosurg 2021;202:106510

13. Starke RM, Snelling B, Al-Mufti F, Gandhi CD, Lee SK, Dabus G, et al.; Society of Neurolnterventional Surgery. Transarterial and transvenous access for neurointerventional surgery: report of the SNIS Standards and Guidelines Committee. J Neurointerv Surg 2020;12:733-741

14. Ramos AD, Sundararajan S, Santillan A, Schwarz JT, Patsalides A. Single arm access venous sinus stenting (SAVeS) technique: technical note. Interv Neuroradiol 2020;26:501-505

15. Abecassis IJ, Saini V, Phillips TJ, Osbun JW, Martínez-Galdámez M, Nada A, et al. Upper extremity transvenous access for neuroendovascular procedures: an international multicenter case series. J Neurointerv Surg 2021;13:357-362 